\title{
Comparison of tolterodine and Solifancine in treatment of female with overactive bladder
}

\author{
Nermeen Mohamed Hefila*, Tamer Mamdouh And el dayem, Hisham Adel Elfazari
}

Department of Obstetrics and Gynecology, Faculty of Medicine, Alexandria University, El Shatby Medical Campus, Alexandria, Egypt

Received: 02 May 2021

Revised: 16 May 2021

Accepted: 17 May 2021

\section{*Correspondence:}

Dr. Nermeen Mohamed Hefila,

E-mail: nermeenptsbasem81@gmail.com

Copyright: (C) the author(s), publisher and licensee Medip Academy. This is an open-access article distributed under the terms of the Creative Commons Attribution Non-Commercial License, which permits unrestricted non-commercial use, distribution, and reproduction in any medium, provided the original work is properly cited.

\section{ABSTRACT}

Background: OAB (overactive bladder) is primarily a neuromuscular problem in which the detrusor muscle contracts inappropriately during bladder filling (i.e., storage phase). These contractions often occur regardless of the amount of urine in the bladder. The aim of this work is to study the effect of Solifenacin in comparison to Tolterodine in treatment of females with idiopathic over active bladder.

Methods: This study included 50 patients who presented between March 2020 and February2021 to the outpatients clinic at El-Shatby maternity Hospital, Alexandria university. The patients were complaining of urinary urgency usually with urinary frequency, nocturia with or without urge incontinence. All cases were divided in two groups one of them receive Solifenacin $5 \mathrm{mg}$ twice daily and the otherreceiveTolterodine $2 \mathrm{mg}$ twice daily.

Results: Both groups showed significant decrease in frequency number per day with $\mathrm{p}$ value $<0.001$ ingroup I with Solifenacin which mean statistically significant difference between two groups. When the percentage of symptoms severity were compared between both groups, group 1 showed 53\% reduction in symptoms severity while group II showed $6.67 \%$ reduction which cleared significant difference statistically in group I ( $\mathrm{p}=0.001)$. The percentage of reduction of severity of symptoms was compared between the two groups, it showed about $60 \%$ reduction in symptoms severity in group 1 while in group II only $9.7 \%$ reduction. This was statistically significant with $(\mathrm{p}<0.001)$ in group I with Solifenacin.

Conclusions: This study suggests that Solifenacin $10 \mathrm{mg} /$ day is more effective than Tolterodine $4 \mathrm{mg} / \mathrm{day}$ in reducing OAB incontinence episodes.

Keywords: Urinary incontinence, Overactive bladder, Tolterodine, Solifenacine

\section{INTRODUCTION}

OAB (overactive bladder) is primarily a neuromuscular problem in which the detrusor muscle contracts inappropriately during bladder filling. These contractions often occur regardless of the amount of urine in the bladder. $\mathrm{OAB}$ may result from a number of different causes, both neurogenic and non-neurogenic. The neurogenic causes are including: spinal cord injury, stroke and neurologic diseases that may cause $\mathrm{OAB}$ include the following: multiple sclerosis and diabetic neuropathy. ${ }^{1}$
Idiopathic $\mathrm{OAB}$ is $\mathrm{OAB}$ in the absence of any underlying neurologic, metabolic, or other causes of $\mathrm{OAB}$, or conditions that may mimic $\mathrm{OAB}$, such as urinary tract infection, bladder cancer, bladder stones, bladder inflammation, or bladder outlet obstruction. More than $90 \%$ of females with detrusor over activity have no other recognizable cause. ${ }^{2}$

According to the international continence society (ICS) definition, $\mathrm{OAB}$ consists of urinary urgency with or without urgency urinary incontinence (UUI), often 
accompanied by frequency and nocturia, in the absence of urinary tract infection (UTI) or other obvious pathology. ${ }^{3}$ OAB symptoms can cause psychological distress, and have a profound negative impact on health related quality of life, relationships, and self-esteem. The ICS defines urinary frequency as the patient complaint of urinating too often during the daytime. The ICS definition of nocturia is one or more voids per night, preceded and followed by sleep. Urgency is the complaint of a sudden, compelling desire to pass urine. Urgency incontinence is the involuntary leakage of urine accompanied or immediately preceded by urgency. ${ }^{3}$

These standard definitions are, in places, controversial. Frequency has conventionally been considered as voiding $\geq 8$ times per 24 hours. $^{3}$

Stress urinary incontinence (SUI) is defined by the international continence society (ICS) as the complaint of involuntary leakage of urine on effort or sudden in intraabdominal pressure ason sneezing or coughing.

A third type of incontinence called mixed incontinence, Detrusor overactivity can coexist with stress incontinence in up to $30 \%$ of patients. Whether this is a coincidental or underlying relationship between these two conditions is unknown. In women with mixed stress and urge incontinence, a deficient urethral sphincter may result in urge incontinence, if leakage of urine into the proximal urethra stimulates urethral afferents that induce involuntary voiding reflexes. Interestingly, after antiincontinence surgery, detrusor over activity may disappear, remain the same, or worsen.

The evaluation should begin with a focused history and questionnaire (OAB) Overactive. The questions ask about how bothered you may be by some bladder symptoms. ${ }^{4}$

A concise history may be obtained using questionnaires providing the patient time to consider her symptoms, past medical history, and family history. Bowel and bladder diaries or other symptoms recorded at home may provide further clarification of the patient's symptoms. During the focused history and questionnaire, the health care provider should ask about LUTS, as well as the presence or absence of: diabetes, neurologic disorders, recurrent UTI, hematuria, kidney stones, previous lower abdominal or pelvic surgery, pelvic organ prolapse, vaginitis. ${ }^{5}$

A urinalysis should be conducted for all patients with $\mathrm{OAB}$ symptoms.

General examination include:

Neurologic, urologic/gynecologic, vaginal exam: During the vaginal exam, the provider should assess perineal sensation and reflexes.

The core test of an urodynamic investigation is cystometry. ${ }^{6}$ Cystometry is the continuous measurement of the pressure/volume relationship of the bladder to assess sensations, detrusor activity, bladder capacity and bladder compliance. $^{7}$

The individual components of urodynamic testing are numerous, following components are most essential to planning surgical therapy: Stress testing, cystometry, uroflowmetry, postvoidresidual urine volume determination, abdominal (or valsalva) leak point pressure. ${ }^{8}$

\section{Treatment}

First-line treatment: Behavioral therapies

International clinical practice guidelines recommend patient education, lifestyle advice and bladder training for the initial management of OAB. ${ }^{9}$

Pelvic floor muscle training (PFMT) is achieved by Kegel exercises which may be aided by biofeedback or electrical stimulation.

\section{Second-line treatment: Pharmacologic management}

\section{Antimuscarinic drugs}

Antimuscarinic drugs act as inhibit involuntary detrusor contractions and thus reduce urgency which could be: Non-selective - have affinity for all muscarinic receptors. Propantheline bromide, tolterodine tartrate, trospium chloride or selective - have relatively more affinity for M2 and M3 muscarinic receptors. Darifenacin, oxybutynin, solifenacine M3 selective antagonist.

\section{Potassium channel openers}

These agents efficiently relax various types of smooth muscle (including detrusor smooth muscle).

\section{Calcium antagonists}

Tricyclic antidepressants

Decreasing sensory input

Systemic and topical capsaicin produces a reversible antinociceptive and anti-inflammatory action.

\section{Therapy to increase outlet resistance}

Including adrenergic agonists medications.

\section{Estrogens for urinary incontinence}

It is most likely through one or more of the following mechanisms: (a) raising the sensory threshold of the bladder and/or urethra; (b) increasing the a -adrenoceptor sensitivity in urethral smooth muscle; (c) increasing 
urethral resistance by (b) or by another mechanism; (d) correcting underlying urogenital atrophy.

Third-line treatment of OAB: Botulinum toxin and Neuro modulationas Sacral nerve stimulation Tibialnerve stimulation The percutaneous posterior tibial nerve stimulation (PTNS)The transcutaneous posterior tibial nerve stimulation (TPTNS).

Surgical treatment: cystoplasty.

\section{Aim}

The aim of this work is to study the effect of Solifenacin in comparison to Tolterodine in treatment of females with idiopathic over-active bladder.

\section{METHODS}

This prospective study was conducted on females patients between 40-60 years old diagnosed as OAB presented to the outpatients clinic of Obstetrics and Gynecology at ElShatby Maternity Hospital from March 2020 till February 2021.

Patients were randomized into 2 groups. Group $1(n=25)$ received $5 \mathrm{mg}$.

Solifenacinper day, while Group $2(n=25)$ received $2 \mathrm{mg}$ Tolterodine per day. All the patients underwent cystometry and the $\mathrm{OAB}$ questionaries in weeks 0 , and 12 weeks after treatment and the results were recorded. In addition, side effects of the drugs were evaluated.

\section{Exclusion criteria}

Females with urinary tract infection, presence of urinary stones, presence of tumor in urinary tract, females having neurogenic voiding dysfunction, known hypersensitivity or contraindication to the drug, female with stress urinary incontinence, female with genital prolapse.

The cases were randomly divided into two equal groups.

\section{Group I (twenty-five women)}

Women in this group were informed about the study and sign an informed consent. They will receive $5 \mathrm{mg}$ Solifenacin 2 times per day.

\section{Group II (twenty-five women)}

These women were received $2 \mathrm{mg}$ Tolterodine 2 times per day.

For all patients' OAB symptom for the beginning week (0) were recorded by $\mathrm{OAB}$ questionnaire and cystometry, and at week 12 after treatment. This study included 50 patients who presented between March 2020 and February2021 to the outpatient's clinic at El-Shatby maternity Hospital, Alexandria university. The patients were complaining of urinary urgency usually with urinary frequency, nocturia with or without urge incontinence. All of the patients were thoroughly investigated.

Full history taking and $\mathrm{OAB}$ questionnaire were done:

Personal history regarding the age, marital status, occupation, address, menstrual status, parity and if there is history of difficult labour.

Past history of other diseases were noted, especially genitourinary diseases including uterine prolapse, stress urinary incontinence, and bladder dysfunction due to neuropathic disease.

History of chronic diseases like diabetes mellitus, hypertension and stroke, also neurological diseases like myelomenegiocele, transverse myelitis and meningitis was taken.

Drug history, history of previous pelvic operations and history of spinal injury were also taken.

We defined patient's complains including frequency, urgency, nocturia, nocturnal enuresis and urge incontinence according the ICS standardization. History of each complain includes its onset, duration, severity and number of each episode per 24 hours was also evaluated. Examination was done in females to detect vaginal atrophy due to estrogen deficiency and pelvic organ prolapse. Perineal examination for perineal defect and weakness. Perineal examination included basic neurological examination (sensation, anal reflexes and anal tone) and lower back examination to detect masses, hair distribution and scars of previous surgeries.

Assessment of pelvic floor function was done by looking at the perineum and asking the patient to contract the pelvic floor and also by vaginal or digital rectal examination. Neurological examination included assessment of sensation, power and reflexes of the legs and the perineum.

Routine laboratory investigations including urine analysis and urine culture were done to exclude patients with urinary tract infection which may be misdiagnosed as $\mathrm{OAB}$.

Plain-x-ray of the urinary tract was done for cases with pervious history of lower urinary tract stones and masses to exclude stones or any bony skeleton diseases.

\section{Cystometry}

One way was connected to a transducer to measure vesical pressure and the other for bladder filling. 
After 3 months of treatment to both groups (group I with Solifenacin $10 \mathrm{mg} /$ day and group II with Tolterodine 4 $\mathrm{mg} /$ day) OAB questionnaire was assed and also cystometery was done to evaluate the difference in symptoms and its severity, also evaluation of the side effects of both medications in both groups was done.

This study was approved ethically by ethics committee for the scientific research. All Institutional and National Guidelines for the care and use of animals (insects) were followed.

\section{Statistical analysis of the data ${ }^{10}$}

Data were fed to the computer and analyzed using IBM Statistical package for social science (SPSS) software package version 20.0. (Armonk, NY: IBM Corp). ${ }^{11}$

\section{RESULTS}

Mean patients age was 50-52 years in both group with no significant difference $(\mathrm{p}=0.28)$. As regards parity and gravidity, both groups were also similar with mean parity 3.

Both groups were similar in the number of pre-treatment of daytime frequency (12-24 time). After treatment, both groups showed significant decrease in frequency number per day (6-8) with $\mathrm{p}<0.001$ in group I with Solifenacin which mean statistically significant difference between two groups.

When the percentage of change in symptoms severity were compared between both groups, group 1 showed 53\% reduction in symptoms severity while group II showed $6.67 \%$ reduction which cleared significant difference statistically in group I ( $\mathrm{p}=0.001)$ (Table 3$)$.

Table 1: Comparison between the two studied groups according to demographic data.

\begin{tabular}{|c|c|c|c|c|}
\hline Age (years) & Group I (n=25) & Group II (n=25) & $\mathbf{t}$ & P value \\
\hline Min-Max & $40.0-58.0$ & $40.0-60.0$ & \multirow{3}{*}{1.075} & \multirow{3}{*}{0.288} \\
\hline Mean \pm SD & $50.76 \pm 4.94$ & $49.16 \pm 5.57$ & & \\
\hline Median & 52.0 & 50.0 & & \\
\hline
\end{tabular}

t: Student t-test, p: p value for comparison between the two studied groups. Group I: Solifenacin (5 mg), Group II: Tolterodine (2 mg)

Table 2: Comparison between the two studied groups according to obstetric data.

\begin{tabular}{|c|c|c|c|c|c|c|}
\hline \multirow{2}{*}{ Obstetric data } & \multicolumn{2}{|c|}{ Group I $(n=25)$} & \multicolumn{2}{|c|}{ Group II $(n=25)$} & \multirow{2}{*}{ Test of sig. } & \multirow{2}{*}{ P value } \\
\hline & No. & $\%$ & No. & $\%$ & & \\
\hline \multicolumn{7}{|l|}{ Gravidity } \\
\hline 2 & 7 & 28.0 & 10 & 40.0 & \multirow{4}{*}{$\begin{array}{l}\chi^{2}= \\
5.773\end{array}$} & \multirow{4}{*}{$\begin{array}{l}{ }^{{ }^{M C}} \mathrm{p}= \\
0.121\end{array}$} \\
\hline 3 & 8 & 32.0 & 8 & 32.0 & & \\
\hline 4 & 5 & 20.0 & 7 & 28.0 & & \\
\hline 5 & 5 & 20.0 & 0 & 0.0 & & \\
\hline Min.-Max. & \multicolumn{2}{|c|}{$2.0-5.0$} & \multicolumn{2}{|c|}{$2.0-4.0$} & \multirow{3}{*}{$\begin{array}{l}U= \\
245.50\end{array}$} & \multirow{3}{*}{0.174} \\
\hline Mean \pm SD. & \multicolumn{2}{|c|}{$3.32 \pm 1.11$} & \multicolumn{2}{|c|}{$2.88 \pm 0.83$} & & \\
\hline Median & \multicolumn{2}{|c|}{3.0} & \multicolumn{2}{|c|}{3.0} & & \\
\hline \multicolumn{7}{|l|}{ Parity } \\
\hline 1 & 2 & 8.0 & 0 & 0.0 & \multirow{5}{*}{$\begin{array}{l}\chi^{2}= \\
5.789\end{array}$} & \multirow{5}{*}{$\begin{array}{l}{ }^{\mathrm{MC}} \mathrm{p}= \\
0.173\end{array}$} \\
\hline 2 & 5 & 20.0 & 10 & 40.0 & & \\
\hline 3 & 8 & 32.0 & 10 & 40.0 & & \\
\hline 4 & 8 & 32.0 & 5 & 20.0 & & \\
\hline 5 & 2 & 8.0 & 0 & 0.0 & & \\
\hline Min.-Max. & \multicolumn{2}{|c|}{$1.0-5.0$} & \multicolumn{2}{|c|}{$2.0-4.0$} & \multirow{3}{*}{$\begin{array}{l}U= \\
250.00\end{array}$} & \multirow{3}{*}{0.203} \\
\hline Mean \pm SD. & \multicolumn{2}{|c|}{$3.12 \pm 1.09$} & \multicolumn{2}{|c|}{$2.80 \pm 0.76$} & & \\
\hline Median & \multicolumn{2}{|c|}{3.0} & \multicolumn{2}{|c|}{3.0} & & \\
\hline
\end{tabular}

$\chi^{2}$ :Chi square tes, MC: Monte Carlo, U: Mann Whitney test, $\mathrm{p}: \mathrm{p}$ value for comparison between the two studied groups. Group I: Solifenacin (5 mg), Group II: Tolterodine ( $2 \mathrm{mg})$.

Both groups were similar in the number of pre-treatment night time frequency around 4times or more per night. After treatment, both groups showed significant improvement with more improvement in group I $(\mathrm{p}<0.001)$.
When the percentage of reduction was compared between the two groups, it showed about $60 \%$ reduction in symptoms severity in group 1while in group II only $9.7 \%$ reduction in symptoms severity was observed. This was statistically significant with $(\mathrm{p}<0.001)$ in group I with Solifenacin (Table 4). 
Table 3: Comparison between the two studied groups according to day time frequency (pre and post treatment).

\begin{tabular}{|c|c|c|c|c|c|c|}
\hline \multirow[t]{2}{*}{ Q1. How often do you usually urinate during the day? } & \multicolumn{2}{|c|}{$\begin{array}{l}\text { Group I } \\
(n=25)\end{array}$} & \multicolumn{2}{|c|}{$\begin{array}{l}\text { Group II } \\
(\mathbf{n}=\mathbf{2 5})\end{array}$} & \multirow[t]{2}{*}{$\chi^{2}$} & \multirow[t]{2}{*}{${ }^{\mathrm{MC}} \mathbf{p}$} \\
\hline & No. & $\%$ & No. & $\%$ & & \\
\hline \multicolumn{7}{|l|}{ Pre treatment } \\
\hline No more often than once in 4 hours & 0 & 0.0 & 0 & 0.0 & \multirow{4}{*}{0.117} & \multirow{4}{*}{0.733} \\
\hline About every 3-4 hours & 0 & 0.0 & 0 & 0.0 & & \\
\hline About every $2-3$ hours & 6 & 24.0 & 5 & 20.0 & & \\
\hline About every 1-2 hours & 19 & 76.0 & 20 & 80.0 & & \\
\hline \multicolumn{7}{|l|}{ Post treatment } \\
\hline No more often than once in 4 hours & 9 & 36.0 & 1 & 4.0 & \multirow{5}{*}{$33.272^{*}$} & \multirow{4}{*}{$<0.001^{*}$} \\
\hline About every 3-4 hours & 13 & 52.0 & 3 & 12.0 & & \\
\hline About every $2-3$ hours & 3 & 12.0 & 4 & 16.0 & & \\
\hline About every $1-2$ hours & 0 & 0.0 & 17 & 68.0 & & \\
\hline${ }^{\mathrm{MH}} \mathrm{p}_{1}$ & \multicolumn{2}{|c|}{$<0.001^{*}$} & \multicolumn{2}{|c|}{0.131} & & \\
\hline
\end{tabular}

$\chi^{2}$ :Chi square test MC: Monte Carlo, MH: Marginal Homogeneity test, $\mathrm{p}$ : $\mathrm{p}$ value forcomparison between the two studied groups, $\mathrm{p} 1: \mathrm{p}$ value for comparison between pre and post treatment, *: Statistically significant at $\mathrm{p} \leq 0.05$, Group I: Solifancine (5 mg),Group II: Tolterodine $(2 \mathrm{mg})$

Table 4: Comparison between the two studied groups according to pre and post treatment of night time frequency.

\begin{tabular}{|c|c|c|c|c|c|c|}
\hline \multirow{2}{*}{$\begin{array}{l}\text { Q2.How many times do you usually urinate at night } \\
\text { (from the time you go to bed until the time you wake } \\
\text { up for the day)? }\end{array}$} & \multicolumn{2}{|c|}{$\begin{array}{l}\text { Group I } \\
(\mathbf{n}=\mathbf{2 5})\end{array}$} & \multicolumn{2}{|c|}{$\begin{array}{l}\text { Group II } \\
(\mathbf{n}=25)\end{array}$} & \multirow[t]{2}{*}{$\chi^{2}$} & \multirow[t]{2}{*}{${ }^{\mathrm{MC}_{\mathbf{p}}}$} \\
\hline & No. & $\%$ & No. & $\%$ & & \\
\hline \multicolumn{7}{|l|}{ Pre treatment } \\
\hline $0-1$ times & 0 & 0.0 & 0 & 0.0 & \multirow{5}{*}{5.472} & \multirow{5}{*}{0.065} \\
\hline 2 times & 0 & 0.0 & 0 & 0.0 & & \\
\hline 3 times & 14 & 56.0 & 8 & 32.0 & & \\
\hline 4 times & 3 & 12.0 & 10 & 40.0 & & \\
\hline 5 or more times & 8 & 32.0 & 7 & 28.0 & & \\
\hline \multicolumn{7}{|l|}{ Post treatment } \\
\hline 0-1 times & 14 & 56.0 & 0 & 0.0 & \multirow{6}{*}{$39.496^{*}$} & \multirow{5}{*}{$<0.001^{*}$} \\
\hline 2 times & 11 & 44.0 & 5 & 20.0 & & \\
\hline 3 times & 0 & 0.0 & 8 & 32.0 & & \\
\hline 4 times & 0 & 0.0 & 9 & 36.0 & & \\
\hline 5 or more times & 0 & 0.0 & 3 & 12.0 & & \\
\hline${ }^{\mathrm{MH}} \mathrm{p}_{1}$ & \multicolumn{2}{|c|}{$<0.001^{*}$} & \multicolumn{2}{|c|}{0.052} & & \\
\hline
\end{tabular}

$\chi 2$ :Chi square test, MC: Monte CarloMH: Marginal Homogeneity test, $\mathrm{p}: \mathrm{p}$ value forcomparison between the two studied groups, $\mathrm{p} 1: \mathrm{p}$ value forcomparison between pre and post treatment, *: Statistically significant at $p \leq 0.05$, Group I: Solifenacin (5mg),Group II:

Tolterodine $(2 \mathrm{mg})$

Table 5: Comparison between the two studied groups regarding pre and post treatment urgency.

\begin{tabular}{|c|c|c|c|c|c|c|}
\hline \multirow[t]{2}{*}{ Q3.What is the reason that you usually urinate? } & \multicolumn{2}{|c|}{$\begin{array}{l}\text { Group I } \\
(\mathbf{n}=\mathbf{2 5})\end{array}$} & \multicolumn{2}{|c|}{$\begin{array}{l}\text { Group II } \\
(\mathbf{n}=\mathbf{2 5})\end{array}$} & \multirow{2}{*}{$\chi^{2}$} & \multirow[t]{2}{*}{${ }^{\mathrm{MC}} \mathbf{p}$} \\
\hline & No. & $\%$ & No. & $\%$ & & \\
\hline \multicolumn{7}{|l|}{ Pre treatment } \\
\hline Out of convenience (no urge or desire) & 0 & 0.0 & 0 & 0.0 & \multirow{5}{*}{0.496} & \multirow{5}{*}{0.849} \\
\hline $\begin{array}{l}\text { Because I have a mild urge or desire (but can delay } \\
\text { urination for over an hour if I have to) }\end{array}$ & 0 & 0.0 & 0 & 0.0 & & \\
\hline $\begin{array}{l}\text { Because I have a moderate urge or desire (but can delay } \\
\text { urination for more than } 10 \text { but less than } 60 \text { minutes if I have } \\
\text { to) }\end{array}$ & 2 & 8.0 & 3 & 12.0 & & \\
\hline $\begin{array}{l}\text { Because I have a severe urge or desire (but can delay urination } \\
\text { for less than } 10 \text { minutes if I have to) }\end{array}$ & 8 & 32.0 & 9 & 36.0 & & \\
\hline $\begin{array}{l}\text { Because I have desperate urge or desire (must stop what I } \\
\text { am doing and go immediately) }\end{array}$ & 15 & 60.0 & 13 & 52.0 & & \\
\hline
\end{tabular}

\section{Post treatment}




\begin{tabular}{|c|c|c|c|c|c|c|}
\hline \multirow[t]{2}{*}{ Q3. What is the reason that you usually urinate? } & \multicolumn{2}{|c|}{$\begin{array}{l}\text { Group I } \\
(\mathbf{n}=\mathbf{2 5})\end{array}$} & \multicolumn{2}{|c|}{$\begin{array}{l}\text { Group II } \\
(\mathbf{n}=25)\end{array}$} & \multirow[t]{2}{*}{$\chi^{2}$} & \multirow[t]{2}{*}{${ }^{\mathrm{MC}} \mathbf{p}$} \\
\hline & No. & $\%$ & No. & $\%$ & & \\
\hline Out of convenience (no urge or desire) & 7 & 28.0 & 4 & 16.0 & \multirow{6}{*}{$10.992^{*}$} & \multirow{5}{*}{0.014} \\
\hline $\begin{array}{l}\text { Because I have a mild urge or desire (but can delay } \\
\text { urination for over an hour if I have to) }\end{array}$ & 17 & 68.0 & 10 & 40.0 & & \\
\hline $\begin{array}{l}\text { Because I have a moderate urge or desire (but can delay } \\
\text { urination for more than } 10 \text { but less than } 60 \text { minutes if I have } \\
\text { to) }\end{array}$ & 1 & 4.0 & 3 & 12.0 & & \\
\hline $\begin{array}{l}\text { Because I have a severe urge or desire (but can delay urination } \\
\text { for less than } 10 \text { minutes if I have to) }\end{array}$ & 0 & 0.0 & 5 & 20.0 & & \\
\hline $\begin{array}{l}\text { Because I have desperate urge or desire (must stop what I } \\
\text { am doing and go immediately) }\end{array}$ & 0 & 0.0 & 3 & 12.0 & & \\
\hline${ }^{\mathrm{MH}} \mathrm{p}_{1}$ & \multicolumn{2}{|c|}{$<0.001^{*}$} & \multicolumn{2}{|c|}{$<0.001^{*}$} & & \\
\hline
\end{tabular}

$\chi^{2}:$ Chi square test, MC: Monte Carlo, MH: Marginal Homogeneity test, $\mathrm{p}: \mathrm{p}$ value for comparison between the two studied groups, $\mathrm{p}_{1}: \mathrm{p}$ value forcomparison between pre and post treatment, *: Statistically significant at $\mathrm{p} \leq 0.05$, Group I: Solifenacin ( $5 \mathrm{mg}), \mathrm{Group}$ II: Tolterodine (2 mg)

Table 6: Comparison between the two studied groups according to pre and post treatment ability to control urgency.

\begin{tabular}{|c|c|c|c|c|c|c|}
\hline \multirow{2}{*}{$\begin{array}{l}\text { Q4.Once you get the urge or desire to urinate, how long } \\
\text { can you usually postpone it comfortably? }\end{array}$} & \multicolumn{2}{|c|}{$\begin{array}{l}\text { Group I } \\
(\mathrm{n}=25)\end{array}$} & \multicolumn{2}{|c|}{$\begin{array}{l}\text { Group II } \\
(\mathrm{n}=\mathbf{2 5})\end{array}$} & \multirow{2}{*}{$\chi^{2}$} & \multirow[t]{2}{*}{$\mathrm{MC}_{\mathbf{p}}$} \\
\hline & No. & $\%$ & No. & $\%$ & & \\
\hline \multicolumn{7}{|l|}{ Pre treatment } \\
\hline More than 60 minutes & 0 & 0.0 & 0 & 0.0 & \multirow{5}{*}{0.399} & \multirow{5}{*}{1.000} \\
\hline About $30-60$ minutes & 0 & 0.0 & 0 & 0.0 & & \\
\hline About $10-30$ minutes & 3 & 12.0 & 2 & 8.0 & & \\
\hline A few minutes (less than 10 minutes) & 5 & 20.0 & 6 & 24.0 & & \\
\hline Must go immediately & 17 & 68.0 & 17 & 68.0 & & \\
\hline \multicolumn{7}{|l|}{ Post treatment } \\
\hline More than 60 minutes & 5 & 20.0 & 5 & 20.0 & \multirow{6}{*}{$11.236^{*}$} & \multirow{5}{*}{$0.015^{*}$} \\
\hline About $30-60$ minutes & 14 & 56.0 & 7 & 28.0 & & \\
\hline About $10-30$ minutes & 3 & 12.0 & 2 & 8.0 & & \\
\hline A few minutes (less than 10 minutes) & 3 & 12.0 & 3 & 12.0 & & \\
\hline Must go immediately & 0 & 0.0 & 8 & 32.0 & & \\
\hline${ }^{\mathrm{MH}} \mathrm{p}_{1}$ & \multicolumn{2}{|c|}{$<0.001^{*}$} & \multicolumn{2}{|c|}{$<0.001^{*}$} & & \\
\hline
\end{tabular}

$\chi^{2}$ :Chi square test, MC: Monte Carlo, MH: Marginal Homogeneity test, $\mathrm{p}: \mathrm{p}$ value for comparison between the two studied groups, $\mathrm{p}_{1}: \mathrm{p}$ value for comparison between pre and post treatment, *: Statistically significant at $\mathrm{p} \leq 0.05$, Group I: Solifenacin (5 mg), Group II: Tolterodine ( $2 \mathrm{mg}$ ).

Table 7: Comparison between the two studied groups regarding the incidence of urge episodes.

\begin{tabular}{|c|c|c|c|c|c|c|}
\hline \multirow{2}{*}{$\begin{array}{l}\text { Q5.How often do you get a sudden urge or desire to } \\
\text { urinate that makes you want to stop what you are doing } \\
\text { and rush to the bathroom? }\end{array}$} & \multicolumn{2}{|c|}{$\begin{array}{l}\text { Group I } \\
(n=25)\end{array}$} & \multicolumn{2}{|c|}{$\begin{array}{l}\text { Group II } \\
(\mathbf{n}=\mathbf{2 5})\end{array}$} & \multirow[t]{2}{*}{$\chi^{2}$} & \multirow[t]{2}{*}{$\mathrm{MC}_{\mathbf{p}}$} \\
\hline & No. & $\%$ & No. & $\%$ & & \\
\hline \multicolumn{5}{|l|}{ Pre treatment } & \multirow{6}{*}{0.117} & \multirow{6}{*}{0.733} \\
\hline Never & 0 & 0.0 & 0 & 0.0 & & \\
\hline Rarely & 0 & 0.0 & 0 & 0.0 & & \\
\hline A few times a month & 0 & 0.0 & 0 & 0.0 & & \\
\hline A few times a week & 5 & 20.0 & 6 & 0.0 & & \\
\hline At least once a day & 20 & 80.0 & 19 & 76.0 & & \\
\hline \multicolumn{7}{|l|}{ Post treatment } \\
\hline Never & 20 & 80.0 & 7 & 28.0 & \multirow{5}{*}{$14.649^{*}$} & \multirow{5}{*}{$<0.001^{*}$} \\
\hline Rarely & 5 & 20.0 & 13 & 52.0 & & \\
\hline A few times a month & 0 & 0.0 & 5 & 20.0 & & \\
\hline A few times a week & 0 & 0.0 & 0 & 0.0 & & \\
\hline At least once a day & 0 & 0.0 & 0 & 0.0 & & \\
\hline${ }^{\mathrm{MH}} \mathrm{p}_{1}$ & \multicolumn{2}{|c|}{$<0.001^{*}$} & \multicolumn{2}{|c|}{$<0.001^{*}$} & & \\
\hline
\end{tabular}

$\chi 2$ :Chi square test, MC: Monte Carlo, MH: Marginal Homogeneity test, $\mathrm{p}: \mathrm{p}$ value for comparison between the two studied groups, $\mathrm{p} 1: \mathrm{p}$ value for comparison between pre and post treatment, *: Statistically significant at $\mathrm{p} \leq 0.05$, Group I: Solifenacin (5 mg), Group II: Tolterodine ( $2 \mathrm{mg}$ ) 
Table 8: Comparison between the two studied groups regarding the effect of urgency on patient daily activity.

\begin{tabular}{|c|c|c|c|c|c|c|}
\hline \multirow{2}{*}{$\begin{array}{l}\text { Q6. How often do you get a sudden urge or desire to } \\
\text { urinate that makes you want to stop what you are doing } \\
\text { and rush to the bathroom but you do not get there in time } \\
\text { (i.e. you leak urine or wet pads)? }\end{array}$} & \multicolumn{2}{|c|}{$\begin{array}{l}\text { Group I } \\
(\mathbf{n}=\mathbf{2 5})\end{array}$} & \multicolumn{2}{|c|}{$\begin{array}{l}\text { Group II } \\
(\mathbf{n}=\mathbf{2 5})\end{array}$} & \multirow{2}{*}{$\chi^{2}$} & \multirow{2}{*}{${ }^{\mathrm{MC}} \mathbf{p}$} \\
\hline & No. & $\%$ & No. & $\%$ & & \\
\hline \multicolumn{5}{|l|}{ Pre treatment } & \multirow{6}{*}{0.089} & \multirow{6}{*}{1.000} \\
\hline Never & 0 & 0.0 & 0 & 0.0 & & \\
\hline Rarely & 0 & 0.0 & 0 & 0.0 & & \\
\hline A few times a month & 0 & 0.0 & 0 & 0.0 & & \\
\hline A few times a week & 8 & 32.0 & 9 & 36.0 & & \\
\hline At least once a day & 17 & 4.0 & 16 & 64.0 & & \\
\hline \multicolumn{5}{|l|}{ Post treatment } & \multirow{7}{*}{$9.668^{*}$} & \multirow{7}{*}{$0.017^{*}$} \\
\hline Never & 7 & 28.0 & 5 & 20.0 & & \\
\hline Rarely & 13 & 52.0 & 5 & 20.0 & & \\
\hline A few times a month & 2 & 8.0 & 10 & 40.0 & & \\
\hline A few times a week & 3 & 12.0 & 5 & 20.0 & & \\
\hline At least once a day & 0 & 0.0 & 0 & 0.0 & & \\
\hline${ }^{\mathrm{MH}} \mathrm{p}_{1}$ & \multicolumn{2}{|c|}{$<0.001^{*}$} & \multicolumn{2}{|c|}{$<0.001^{*}$} & & \\
\hline
\end{tabular}

$\chi 2$ :Chi square test, MC: Monte Carlo, MH: Marginal Homogeneity test, $\mathrm{p}$ : $\mathrm{p}$ value for comparison between the two studied groups, $\mathrm{p} 1: \mathrm{p}$ value for comparison between pre and post treatment, *: Statistically significant at $\mathrm{p} \leq 0.05$, Group I: Solifenacin (5 mg),Group II: Tolterodine ( $2 \mathrm{mg})$

Table 9: Comparison between the two studied groups regarding patient perception about bladder control.

\begin{tabular}{|c|c|c|c|c|c|c|}
\hline \multirow[t]{2}{*}{ Q7. In your opinion how good is your bladder control? } & \multicolumn{2}{|c|}{$\begin{array}{l}\text { Group I } \\
(\mathbf{n}=\mathbf{2 5})\end{array}$} & \multicolumn{2}{|c|}{$\begin{array}{l}\text { Group II } \\
(\mathrm{n}=\mathbf{2 5})\end{array}$} & \multirow[t]{2}{*}{$\chi^{2}$} & \multirow[t]{2}{*}{${ }^{\mathrm{MC}} \mathbf{p}$} \\
\hline & No. & $\%$ & No. & $\%$ & & \\
\hline \multicolumn{5}{|l|}{ Pre treatment } & \multirow{5}{*}{0.085} & \multirow{5}{*}{1.000} \\
\hline Perfect control & 0 & 0.0 & 0 & 0.0 & & \\
\hline Good & 0 & 0.0 & 0 & 0.0 & & \\
\hline Poor & 9 & 36.0 & 10 & 40.0 & & \\
\hline No control at all & 16 & 64.0 & 15 & 60.0 & & \\
\hline \multicolumn{5}{|l|}{ Post treatment } & & \\
\hline Perfect control & 5 & 20.0 & 3 & 12.0 & \multirow{5}{*}{1.234} & \multirow{5}{*}{0.536} \\
\hline Good & 15 & 60.0 & 14 & 56.0 & & \\
\hline Poor & 5 & 20.0 & 8 & 32.0 & & \\
\hline No control at all & 0 & 0.0 & 0 & 0.0 & & \\
\hline${ }^{\mathrm{MH}} \mathrm{p}_{1}$ & \multicolumn{2}{|c|}{$<0.001^{*}$} & \multicolumn{2}{|c|}{$<0.001^{*}$} & & \\
\hline
\end{tabular}

$\chi 2$ :Chi square test, MC: Monte Carlo, MH: Marginal Homogeneity test, $\mathrm{p}: \mathrm{p}$ value forcomparison between the two studied groups, $\mathrm{p} 1: \mathrm{p}$ value forcomparison between pre and post treatment, *: Statistically significant at $\mathrm{p} \leq 0.05$, Group I: Solifenacin (5 mg), Group II:

Tolterodine $(2 \mathrm{mg})$

When both groups were compared regarding urgency before treatment, no difference between both groups was found and mean gravidity 3. (Table 1 and 2). After 3 months, treatment showed improvement in urgency (decrease episodes) in both groups. In group I $61.5 \%$ reduction in severity was detected while group II $40.6 \%$ reduction was found with significant difference in between $\mathrm{p}<0.001$ (Table 5). Both groups were compared regarding the ability to control their urgency. Both were similar before treatment with no difference.

But after treatment, significant control in both groups with $(p<0.001)$ in both group when was calculated the percentage of change in symptoms severity between both groups $4.5 \%$ reduction in symptoms severity was observed in group I, while in group II only $2.6 \%$ reduction of symptoms severity was found. However, there was no statistically significant difference between both group. $(\mathrm{p}=0.041)$ (Table 6).

As regards urgency episodes that cannot be delayed by the patient, both groups were similar before treatment with $p$ value (0.733)

After 3 months treatment, the change in urgency episodes and improvement were more evident in group I $(\mathrm{p}<0.001)$ versus in group II it may happen few times per month but still with significant improvement in group II $(\mathrm{p}<0.001)$.

Comparing the percentage of improvement in urgency. In group 1 while reduction in symptoms severity $74.6 \%$ was found while in group II reduction in symptoms severity 
was $59.6 \%$ with $\mathrm{p}<0.001$ which showed statistically significant between both groups. (Table 7).

Comparing the two groups as regard the effect of urgency on the patients daily life activity before treatment, both groups were equally affected with no statistically significant difference $(\mathrm{p}=1.0)$. After 3 months treatment, improvement in both groups was observed with more improvement in group I (with Solifencien) $(\mathrm{p}<0.001)$ which means statistically significant difference between both groups. While when we compared the percent of change before and after treatment, there was a reduction in symptoms severity in both groups. In group I reduced symptoms was $56.6 \%$ while group II was $45.4 \%$. Meaning significant difference between both of them was detected $(\mathrm{p}=0.015)($ Table 8$)$.

As regards patient perception about bladder control comparing the two groups before treatment, both were equal with no significant difference in between them $(\mathrm{p}=1.000)$. After 3 months of treatment, group 1 showed more improvement in perception of bladder control with $(\mathrm{p}<0.001)$ with statistically significant difference. When were compared the percent of change of perception in both groups, more control in the bladder was found in group I (45.67 \pm 14.66$)$ while in group II percent of change was $(39.33 \pm 14.34)$ with no significant difference between them. $(\mathrm{p}=0.132)$ (Table 9).

Table 10: Comparison between the two studied groups according to side effect.

\begin{tabular}{|c|c|c|c|c|c|c|}
\hline \multirow[t]{2}{*}{ Side effect } & \multicolumn{2}{|c|}{$\begin{array}{l}\text { Group I } \\
(n=25)\end{array}$} & \multicolumn{2}{|c|}{$\begin{array}{l}\text { Group II } \\
(\mathbf{n}=\mathbf{2 5})\end{array}$} & \multirow[t]{2}{*}{$\chi^{2}$} & \multirow[t]{2}{*}{${ }^{\mathrm{FE}} \mathbf{p}$} \\
\hline & No. & $\%$ & No. & $\%$ & & \\
\hline \multicolumn{7}{|l|}{ Dry mouth } \\
\hline None & 23 & 92.0 & 18 & 72.0 & \multirow{2}{*}{3.388} & \multirow{2}{*}{0.138} \\
\hline Yes & 2 & 8.0 & 7 & 28.0 & & \\
\hline \multicolumn{7}{|c|}{ Constipation } \\
\hline None & 20 & 80.0 & 23 & 92.0 & \multirow{2}{*}{1.495} & \multirow{2}{*}{0.417} \\
\hline Yes & 5 & 20.0 & 2 & 8.0 & & \\
\hline \multicolumn{7}{|c|}{ Tachycardia } \\
\hline None & 23 & 92.0 & 20 & 80.0 & \multirow{2}{*}{1.495} & \multirow{2}{*}{0.417} \\
\hline Yes & 2 & 8.0 & 5 & 20.0 & & \\
\hline
\end{tabular}

$\chi 2$ :Chi square test, FE: Fisher Exact test, $\mathrm{p}$ : $\mathrm{p}$ value for comparison between the two studied groups, Group I: Solifenacin (5 mg), Group II: Tolterodine $(2 \mathrm{mg})$

The result of urodynamic were analyzed before and after treatment. Comparing both groups as regards bladder capacity before treatment, mean capacity was $(254.4 \pm 68.09 \mathrm{ml})$ while in group II $(362.4 \pm 110.6 \mathrm{ml})$.After 3 months treatment, there was significant increase in bladder capacity in group I (with Solifenacin) mean capacity $305.8+65.04 \mathrm{ml})$ while in group II $(387.0 \pm 94.12$ $\mathrm{ml}$ ) (with Tolterodine) with $\mathrm{p}=0.001$ which is a statistically significant.

The percent of change in capacity was $22.3 \pm 13 \%$ versus $9.5 \pm 11 \%(\mathrm{p}=0.001)$.

Urine leak during filling cystometery was compared between both groups before treatment, it was equally present in both groups $(\mathrm{p}=0.2)$. After 3 months treatment, the reduction of urine leak in group I (with Solifencine) was more evident than in group II (with Tolterodine) showing significant difference statistically $(\mathrm{p}=0.04)$.

Finally, the adverse effects were compared between both groups after treatment (dry mouth, constipation, and tachycardia).

It was found that incidence of dry mouth and tachycardia was higher in Tolterodine group than with Solifenacin but with no statistically significant difference ( $p$ value 0.138 0.417). On the other hand, the Solifenacin group showed more incidence of constipation. However, this did not show statistically significant value $(\mathrm{p}=0.417)$. (Table 10)

\section{DISCUSSION}

$\mathrm{OAB}$ consists of urinary urgency with or without urinary incontinence (UUI), often accompanied by frequency and nocturia. It has a profound negative impact on health related quality of life, relationships, and self-esteem. ${ }^{3}$

Overactive bladder is a common disease among females. About I0-15\% of adult women have idiopathic OAB and $5-10 \%$ experience urge incontinence. ${ }^{12}$

The major finding in our study is that both medications were effective in reducing $\mathrm{OAB}$ symptoms in micturation frequency, night-time frequency urgency and incontinence episodes. According to day-time frequency the efficacy appeared more in group I with Solifenacin. An efficacy appeared more in group I with Solifenacin. About $53 \%$ reduction in symptoms severity in group I while in group II only $6.6 \%$ reduction in symptoms severity was observed. This was statistically significant with $(\mathrm{p}<0.001)$. 
Control of urgency was more significant statistically in group I than group II, with significant difference $(p=0.014)$. The effect of urgency on daily life activities was improved in both group but more statistically significant improved in group I (with Solifenacin) with significant difference $(\mathrm{p}=0.017)$. Perception of bladder control was equal in both groups with no significant difference in between both of them.

According to the calculated total score of OAB questionnaire, there was reduction in both group, which means improvement in bladder control .More bladder control and improvement was significant with group I, (with Solifenacin) were mean score $(12.36 \pm 2.9)$ while in group II (with Tolterodine) showed (19.4 \pm 4.16 score) $\mathrm{p}<0.001$.

According to a study published in journal of the Formosan medical association in 2010 included that both medications were equally effective in reducing $\mathrm{OAB}$ symptoms as (frequency of micturation, urgency episodes and incontinence) with no difference, which is not in agreement with our study. This, may be because some patient did not compliance well with medication. Also the questionnaire is a subjective method depending on patient's expectations.

Another meta analyses published in 2018suggested that Solifenacin $5 \mathrm{mg} /$ day is more effective than Tolterodine $4 \mathrm{mg} /$ day in the reducing of incontinence and UUI episodes in adults with $\mathrm{OAB} .{ }^{13}$

Madhuvrata et al, reported significantly fewer leakage episodes/24h and urgency episodes/24h with Solifenacin 5 $\mathrm{mg}$ /day versus Tolterodine ER or IR $4 \mathrm{mg} /$ day. ${ }^{14}$ The other meta-analysis, showed significant benefits from Solifenacin compared with Tolterodine ER or IR $4 \mathrm{mg} /$ day in micturation $/ 24 \mathrm{~h}$, urgency episodes $/ 24 \mathrm{~h}$, and incontinence episodes/24h, both meta-analysis showed that Solifenacin $10 \mathrm{mg} /$ day was more effective than Solifenacin $5 \mathrm{mg} /$ day in reducing micturation/24h.

In a study to compare the efficacy of Solifenacin and Tolterodine published Hallaz et al, 2017 in Northern international medical journal. ${ }^{15}$ The author found that Solifenacin reduced urinary frequency by $(38.82 \pm 9.32 \%)$ while Tolterodine was $(25.85 \pm 10.75 \%)$. In Solifenacin group mean $( \pm \mathrm{SD})$ reduction of frequency of nocturia from base to 3 months after treatment was $(72.75 \pm 21.02)$, and was $(47.08 \pm 12.17)$ in Tolterodine group. The improvement of frequency and nocturia with Solifenacin is statistically significant $(\mathrm{p}<0.001)$. The result reflect superiority of Solifenacin over Tolterodine in reducing frequency and nocturia, which is similar to result of other studies conducted by Chapple et al. ${ }^{16}$

The major findings were significant increase in bladder compliance in group I more than group II. This may indicate that this improvement in compliance is due to the effect of Solifenacin drug rather than the effect of Tolterodine.

The cystometric capacity of bladder increased significantly after treatment and the increase was more in group I than group I1. (377.8 $\pm \mathrm{I} 12.92 \mathrm{ml})$ in group II versus (320.4 $\pm \mathrm{I} 62.3 \mathrm{ml}$ in group I). This increase was near to that in a study done by Klinger et al, which reported an increase from $\mathrm{I} 97 \mathrm{ml}$ to $252 \mathrm{ml} .{ }^{17}$ This is also in agreement with Ammi et al, who mentioned that the cystometric capacity is predictive for success. ${ }^{18}$

Detrusor overactivity showed significant reduction in patient in group I with Solifenacin more than with group II with Tolterodine. This is in agreement with a study done by Vanoninck et al, which stated that DO may not disappear after treatment but if it persists, it is delayed in onset. $^{19}$

Reduction in the occurrence of urine leak was more with group I versus group II. (24 case before treatment and 10 case in group I versus group II (22 case before treatment and $\mathrm{I} 7$ case after treatment) significant reduction in group I $(\mathrm{p}<0.001)$.

Nazir et al, 2018 suggested that both Solifenacin and Tolterodine are well tolerated. In RCT done in 2000-2015 and published in May 2017. ${ }^{13}$ The authors found that Solifenacin $5 \mathrm{mg}$ /day had a lower risk of dry mouth than Tolterodine IR $4 \mathrm{mg} /$ day, and Solifenacin $10 \mathrm{mg} /$ day, and the risk of constipation also did not differ significantly between Solifenacin $5 \mathrm{mg} /$ day (11 of 17 compatator antimuscarinic drugs) was associated with a significantly higher risk of dry mouth. Although the rates of constipation was similar with both doses in one metaanalysis.

Overall Persistence with $\mathrm{OAB}$ medication in clinical practice is challenging for the management of OAB. Treatment discontinuation can be related to several factors, including inadequate drug efficacy, dosing frequency, intolerable adverse effects, patient expectations, and cost. However, Adverse effects associated with antimuscarinic dry mouth, often leads to discontinuation of therapy. ${ }^{20}$ Improvements in incontinence outcomes achieved by switching between antimuscarinic may be minimal. ${ }^{21}$ Selecting the drug that offers the best balance of efficacy and tolerability is an important step in the treatment of patients with $\mathrm{OAB} .^{22}$

\section{CONCLUSION}

This study suggests that Solifenacin $10 \mathrm{mg} /$ day is more effective than Tolterodine $4 \mathrm{mg} /$ day in reducing $\mathrm{OAB}$ incontinence and UUI episodes. Solifenacin $10 \mathrm{mg} /$ day has a lower risk of dry mouth compared with Tolterodine $4 \mathrm{mg}$.

\author{
Funding: No funding sources \\ Conflict of interest: None declared
}


Ethical approval: The study was approved by the Institutional Ethics Committee

\section{REFERENCES}

1. Blok BF, Willemsen AT, Holstege G. A PET study on brain control of micturition in humans. Brain. 1997;120( Pt 1):111-21.

2. Osman NI, Chapple CR. Overactive bladder syndrome: Current pathophysiological concepts and therapeutic approaches. Arab J Urol. 2013;11(4):3138.

3. Abrams P, Cardozo L, Fall M, Griffiths D, Rosier P, Ulmsten $U$, et al. The standardisation of terminology in lower urinary tract function: report from the standardisation sub-committee of the International Continence Society. Urology. 2003;61(1):37-49.

4. Shaw C, Matthews RJ, Perry SI, Williams K, Spiers $\mathrm{N}$, Assassa RP, et al. Validity and reliability of a questionnaire to measure the impact of lower urinary tract symptoms on quality of life: the Leicester Impact Scale. Neurourol Urodyn. 2004;23(3):229-36.

5. Lucas MG, Bedretdinova D, Berghmans LC, Bosch JL, Burkhard FC, Cruz F, et al. Guidelines onurinary incontinence. European Association of Urology. 2015. Available from: https://uroweb.org/wpcontent/uploads/EAU-Guidelines-Urinary-

Incontinence-2015.pdf. Accessed on 20th October 2019.

6. Patel BN, Kobashi KC. Practical use of the new American Urological Association adult urodynamics guidelines. Curr Urol Rep. 2013;14(3):240-6.

7. Cooper MA, Fletter PC, Zaszczurynski PJ, Damaser MS. Comparison of air-charged and water-filled urodynamic pressure measurement catheters. Neurourol Urodyn. 2011;30(3):329-34.

8. Digesu GA, Khullar V, Cardozo L, Salvatore S. Overactive bladder symptoms: do we need urodynamics? Neurourol Urodyn. 2003;22(2):105-8.

9. Cheung WW, Khan NH, Choi KK, Bluth $\mathrm{MH}$, Vincent MT. Prevalence, evaluation and management of overactive bladder in primary care. BMC Fam Pract. 2009;10:8.

10. Kotz S, Balakrishnan N, Read CB, Vidakovic B. Encyclopedia of statistical sciences. Hoboken, N.J.: Wiley-Interscience. 2006.

11. Kirkpatrick LA, Feeney BC. A simple guide to IBM SPSS statistics for version 20.0. Student ed. Belmont, Calif.: Wadsworth, Cengage Learning. 2013.

12. Milsom I, Stewart W, Thuroff J. The prevalence of overactive bladder. Am J Manag Care. 2000;6(11 Suppl):S565-73.

13. Nazir J, Kelleher C, Aballea S, Maman K, Hakimi Z, Mankowski $\mathrm{C}$, et al. Comparative efficacy and tolerability of solifenacin $5 \mathrm{mg} /$ day versus other oral antimuscarinic agents in overactive bladder: A systematic literature review and network metaanalysis. Neurourol Urodyn. 2018;37(3):986-96.

14. Madhuvrata P, Cody JD, Ellis G, Herbison GP, HaySmith EJ. Which anticholinergic drug for overactive bladder symptoms in adults. Cochrane Database Syst Rev. 2012;1:Cd005429.

15. Hallaz MM, Yasmeen B, Sultana N, Akter M, Basher N. Comparison of the efficacy of Solifenacin Succinate and Tolterodine for the treatment of overactive bladder syndrome. NIMCJ. 2017;8(2):2314.

16. Chapple CR, Martinez-Garcia R, Selvaggi L, ToozsHobson P, Warnack W, Drogendijk T, et al. A comparison of the efficacy and tolerability of solifenacin succinate and extended release tolterodine at treating overactive bladder syndrome: results of the STAR trial. Eur Urol. 2005;48(3):464-70.

17. Klingler HC, Pycha A, Schmidbauer J, Marberger M. Use of peripheral neuromodulation of the $\mathrm{S} 3$ region for treatment of detrusor overactivity: a urodynamicbased study. Urology. 2000;56(5):766-71.

18. Ammi M, Chautard D, Brassart E, Culty T, Azzouzi AR, Bigot P. Transcutaneous posterior tibial nerve stimulation: evaluation of a therapeutic option in the management of anticholinergic refractory overactive bladder. Int Urogynecol J. 2014;25(8):1065-9.

19. Vandoninck V, van Balken MR, Finazzi Agro E, Petta F, Micali F, Heesakkers JP, et al. Percutaneous tibial nerve stimulation in the treatment of overactive bladder: urodynamic data. Neurourol Urodyn. 2003;22(3):227-32.

20. Basra R, Basra H, Khullar V, Kelleher C. Prescribiing antimuscarinics for overactive bladder; how many chances do we have to get it right? Int Urogynecol J. 2010;21:S139-40.

21. Chancellor MB, Yehoshua A, Waweru C, Globe D, Cheng IN, Campbell KL, et al. Limitations of anticholinergic cycling in patients with overactive bladder $(\mathrm{OAB})$ with urinary incontinence (UI): results from the CONsequences of Treatment Refractory Overactive bLadder (CONTROL) study. Int Urol Nephrol. 2016;48(7):1029-36.

22. Athanasopoulos A, Giannitsas K. An overview of the clinical use of antimuscarinics in the treatment of overactive bladder. Adv Urol. 2011;2011:820816.

Cite this article as: Hefila NM, el dayem TMA, Elfazari HA. Comparison of tolterodine and Solifancine in treatment of female with overactive bladder. Int J Reprod Contracept Obstet Gynecol 2021;10:2138-47. 